\title{
The Impact of Microcell Deployment on Power Consumption in Heterogeneous Cellular Network
}

\author{
Jie Zhou ${ }^{a}$, Yong Zhang ${ }^{b}$, Mei Song ${ }^{c}$ and Qun Gu ${ }^{d}$
}

School of Electronic Engineering, Beijing University of Posts and Telecommunications, Beijing 100876, China.

achengwai@bupt.edu.cn, byongzhang@bupt.edu.cn, 'songm@bupt.edu.cn, dguqun@bupt.edu.cn

\begin{abstract}
In recent years, people pay more attention to the problem of increasing power consumption of wireless network due to the rapid development of information and communication industry. Using heterogeneous network has been a possible way to solve this problem and improve energy efficiency. Significant research has been conducted. However, most of them only focused on the power consumption of base stations while ignoring the power consumption of mobile devices. In this paper, we start our research with accurate models for both macro and micro base stations and take the power consumption of mobile devices into account. We observe that area power consumption decreases with the increase in the number and radiuses of microcells. Finally, we find it is possible to choose a certain number and radius of microcells to minimize the area power consumption. We verify the correctness of the analysis with simulation.
\end{abstract}

Keywords: Heterogeneous Network; microcell deployment; mobile devices.

\section{Introduction}

In recent years, global warming has becoming a topical issue in global affairs. Information and communication technology (ICT) industry is considered to be a large contributor to the world wide greenhouse gas emissions with its explosive growth. With the deployment of $4 \mathrm{G}$ or $5 \mathrm{G}$ networks, the number of global mobile devices increase rapidly which will lead to more power consumption. Therefore, there is a economic motivation for network operators to reduce power consumption apart from the social responsibility. Many solutions have been proposed in the literature to reduce the power consumption. Heterogeneous cellular networks (HetNets), as one of the approaches to deal with the surging traffic demand and increasing power consumption, has been widely studied. It has been proved in [1] that HetNets can provide high-speed data rates and reduce the transmit power. The mixed deployment scenarios of macrocells and microcells have been investigated in [2]. Simulations have shown that it can reduce the total power consumption by up to $60 \%$ for high data rate user demand in urban areas. Literature [3] analyze the relationship between the power consumption and the quality of service (QoS) support level. The results show that the network deployment combining with microcells not only offers high QoS but also reduces the power consumption. However, most of researches only consider power consumption of the Base Stations (BSs) while ignoring the influence of Mobile Devices (MDs). In fact, there will be more mobile devices in the future. Therefore, power consumption reduction of MDs is becoming more and more important. In [4], several energy efficiency metrics have been introduced for the component, equipment and system level. In this paper, we apply the metric area power consumption defined by Richter in [5]. In this paper, we focus on the effect of microcell deployment strategy on power consumption of HetNets. We apply the detailed power consumption models proposed by [6] to carry on the research. The power consumption of a base station consists of two parts. One part describles the static power consumption, a power figure which is consumed in an idle base station. Depending on the load situation, a dynamic power consumption part adds to the static power. We analyze the power consumption of both BSs and MDs in HetNets. We observe that either increasing the number of microcells or expanding the radius of microcells could reduce the area power consumption under certain conditions. The remainder of the paper is organized as follows. In Section II, our system model and the power consumption metric are 
introduced. In Section III, the analyses of power consumption are provided and in Section IV we verify ouranalysis with extensive simulations. Section V concludes the paper.

\section{System model}

In this section, we firstly present the propagation model. Then, the power consumption models of macro base stations, micro base stations and mobile devices will be introduced. Finally, we describe the metric applied for evaluating the power consumption of HetNets.

\subsection{Propagation Model}

In this paper, we use the path loss model in [7], which has considered the path loss and slow fading. The signal propagation model is formulated as

$$
P_{r x}=K\left(\frac{r}{r_{0}}\right)^{-\lambda} \square P_{t x},
$$

Where $P_{t x}$ the transmission is power of the BS and $P_{r x}$ denotes the received power. $\mathrm{r}$ and $\lambda$ denote the propagation distance and path loss exponent. The parameter $\mathrm{r}_{0}$ specifies a reference distance where signal intensity is known. Its value is $1 \mathrm{~m}$ in this paper. $\mathrm{K}$ denotes a dimensionless constant which depends on antenna characteristics and channel attenuation.

\subsection{Area Power Consumption}

Usually, considering the mere power consumption is not rigorous for comparing cells of different size since larger coverage requires larger transmit power which leads to more power consumption. In order to evaluate the power consumption of the network with respect to its size, we use the notion of area power consumption. It is defined in [5] as

$$
\mathrm{P}_{P / A}=\frac{\text { Total power consumed by all base stations and mobile devices }}{\text { The corresponding cell area }}
$$

In order to calculate the area power consumption, we need to model power consumption behaviour of base stations and mobile devices which are provided in the following section.

\subsection{Power Consumption Models}

The power consumption of a base station includes not only the transmit power but also cooling consumption, power supply loss and signal processing consumption, etc. In fact, cooling consumption accounts for a large proportion of the total consumption. Conventional macro base station which consumes a lot of energy is applied to provide a larger coverage area under the limitation of minimum received power. Micro base station consumes less power and provide a smaller coverage area. In consideration of different features, the model of macro and micro base stations are different.

1) Macrocell Power Consumption: For macro base stations, the dynamic power consumption can be neglected compared with the total power consumption which is proved in [6]. So the model of conventional macro base station can be simplified to the static part. For modeling the power consumption, it is given as

$$
P_{B S, M a}=N_{\mathrm{Sec}} N_{P A}\left(\frac{P_{t x}}{\mu_{P A}}+P_{S P}\right)\left(1+C_{C}\right)\left(1+C_{P S}\right)
$$

Where $P_{B S, M a}$ and $P_{t x}$ denote the total consumed power and transmitted power, $N_{\text {Sec }}$ and $N_{P A}$ denote the number of sectors per base station and the number of power amplifiers per sector, $\mu_{P A}$ denotes the amplifier efficiency, $P_{S P}$ denotes the signal processing overhead, $C_{C}$ denotes the cooling consumption and $C_{P S}$ denotes the battery backup and power supply loss. The values of these parameters which are derived from practical applications are given in Table I.

2) Microcell Power Consumption: Compared with the macro base station, the micro base station has a smaller power consumption and transmit power due to the smaller coverage area. As mentioned above, the power consumption of macro base station is usually independent of traffic load. In contrast, a major advantage of micro base station is its ability to measure its power consumption with the 
current level of activity. In [6], the power consumption of the micro base station consists of two parts: static part and dynamic part. The static part describes the power consumption when it is an idle base station which does not serve to any active user. Dynamic part is the power consumption which depends on the instantaneous load. For modeling the power consumption, the following formula is applied:

$$
P_{B S, M i}=P_{s t a, M i}+P_{d y n, M i},
$$

The static power consumption usually contains the static signal processing power, the power supply loss and the transmit power when there is no active user. We derive the formula as

$$
P_{s t a, M i}=\left(\frac{P_{t x}}{\mu_{P A}} C_{T X, s t a}+P_{S P, s t a}\right)\left(1+C_{P S}\right),
$$

Where $C_{T X, s t a}$ the percentage of is transmit power that is consumed when the base station is idle, $\mu_{P A}$ denotes the decreased power amplifier efficiency compared with the power amplifier of a macro base station and $P_{S P, s t a}$ is the static signal processing power. Considering the size of the microcell and the instantaneous traffic load which cannot be ignored, the dynamic power consumption is given as follows

$$
P_{d y n, M i}=\left(\frac{P_{t x}}{\mu_{P A}}\left(1-C_{T X, s t a}\right) \llbracket C_{t x, N L}+P_{s p, N L}\right) N_{L}\left[\left(1+C_{P S}\right),\right.
$$

Where NL is the number of active links in the microcell $C_{t x, N L}$ denotes the percentage of dynamic transmit power per active link and $P_{s p, N L}$ is dynamic signal processing power for each active link. The values of these parameters are given in Table I.

3) Mobile Devices Power Consumption: Based on the model in [8], the power consumption of MDs is given as

$$
P_{M D}=p P_{\text {idle }}+(1-p) P_{\text {work }} \text {, }
$$

Where $P_{\text {idle }}$ denotes the power consumption when the mobile device is not active and $P_{\text {work }}$ denotes the power consumption when the mobile device is working. The $p$ denotes the probability that the mobile device is not active. The values of these parameters are given in Table I.

\section{Cell deployment and power consumption calculation}

\subsection{Cell Deployment}

In this paper, we investigate the power consumption of two scenarios: one is the macrocell only deployment, the other is the macrocell whose edge is deployed with several microcells. In both scenarios, we keep the coverage fixed which means $95 \%$ of the hexagonal area in Fig.1 is covered either by a macrocell or several microcells. The mobile devices are uniformly distributed in the hexagonal. Firstly, we observe the power consumption of a macrocell only scenario, which is used as a comparison point. Then some microcells are deployed around the macro base station. We assume that the active MDs need to download a data package with size of $\mathrm{S}$ in a unit time (T) which is 1 second. We further assume that both macro base stations and the static part of micro base stations always work at saturation power and the dynamic part of micro base stations depends on the instantaneous traffic load. In this paper, the dynamic part depends on the downloading time of the data package S. Besides, the multicarrier deployment strategy is applied in this paper. The whole spectrum resources are divided into two parts without overlapping. One is utilized by the microcell and the other is for the macrocell. We also assume that all of the MDs at different carrier frequencies can be assigned to the same bandwidth. Thus, the MDs will not interfere with each other. The noise power is $\sigma^{2}$ in this work. So the Signal to Noise Ratio (SNR) at a mobile device is 


$$
\operatorname{SNR}(r)=\frac{P_{r x}}{\sigma^{2}}=\frac{K \square P_{t x} \sqsubset r^{-\lambda}}{\sigma^{2}},
$$

Where $r$ is the distance from the MD to either macro base station or micro base station and Prx denotes the received power. The transmission rate of mobile devices in the cell obey Shannon theorem. It is given as Rate $=B \sqcap \log _{2}(1+S N R)$, where B denotes the bandwidth for each mobile device.

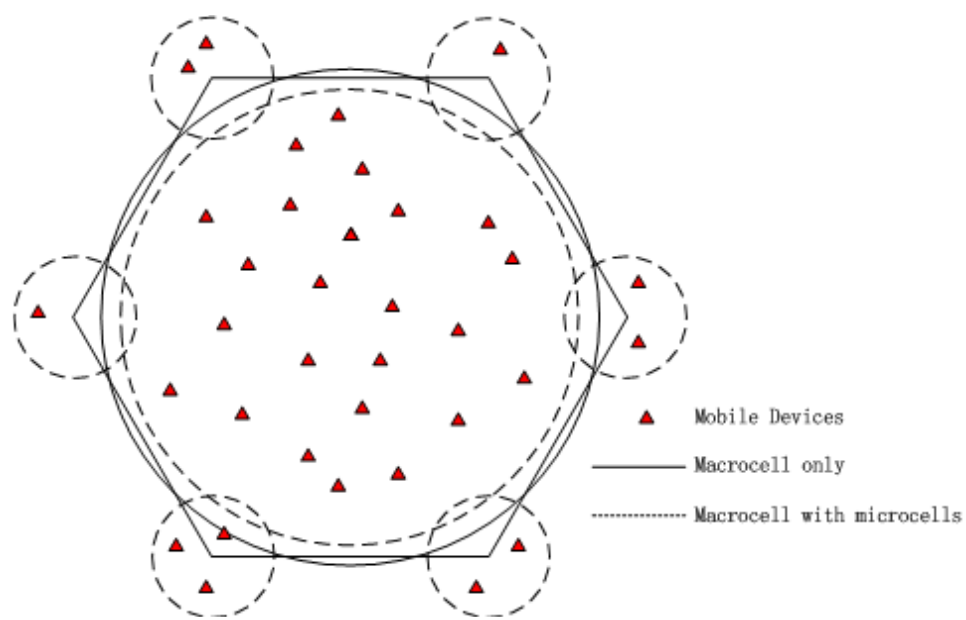

Fig. 1 The deployment scenarios

\subsection{Power Consumption Calculation}

In this part, we will calculate the downloading time and total power consumption of two scenarios. The mobile devices are uniformly distributed throughout the area.

1) Macrocell Only Scenario: Under macrocell only scenario, we assume that the number of mobile devices is $\mathrm{N}$ and the total bandwidth of macro cell is BMA. Therefore, bandwidth of each mobile device in macrocell is

$$
B_{M A, u s e r}=\frac{B_{M A}}{N}
$$

According to the Shannon theorem mentioned above, if SNRR is the minimum SNR required at the edge of macrocell, the transmit power of macrocell is

$$
P_{t x, M A}=S N R_{R} \sqsubset \sigma^{2} \square K^{-1} \square R^{\lambda},
$$

Where R denotes the radius of macrocell. Then we can derive the SNR of mobile device which is $\mathrm{r}$ meters away from base station.

$$
\operatorname{SNR}(r)=\frac{K \square P_{t x, M A} \square^{-\lambda}}{\sigma^{2}}=\operatorname{SNR}_{R}\left(\frac{R}{r}\right)^{\lambda},
$$

According to the Shannon theorem, the transmission rate could be derived as

$$
R_{M A}(r)=B_{M A, u s e r} \operatorname{Dog}_{2}(1+S N R(r)) \text {, }
$$

So the transmission duration of a mobile device at a distance of $\mathrm{r}$ meters from the base station is

$$
t_{M A}(r)=\frac{S}{R_{M A}(r)} \text {, }
$$

In combination with the previous formulas, we derive the average transmission duration of all mobile devices

$$
T_{M A}=\frac{\sum_{i=1}^{N_{M A}} t_{M A}\left(r_{i}\right)}{N},
$$

The total power consumption of mobile devices is 


$$
P_{M D, t o t a l}=N\left[P _ { \text { work } } \left[T_{M A}+P_{i d l e}\left[\left(1-T_{M A}\right)\right]\right.\right. \text {, }
$$

Finally, the total power consumption of macrocell equals to the sum of power consumption of macrocell and all mobile devices:

$$
P_{M A, \text { total }}=P_{B S, \text { Macro }}+P_{M D, \text { total }} \text {. }
$$

2) Macrocell with Microcells Scanario: In this part, because of the addition of microcells, the calculation becomes more complicated. As mentioned above, the macro base stations and the static part of the micro base stations have been always working at full load, while the dynamic part of the micro base stations depends on the downloading time. We assume that there are $\mathrm{N}_{\text {micro }}$ microcells at the edge of macrocell and the number of mobile devices in each microcell is $\mathrm{N}_{\mathrm{i}, \text { Micro }}$ where parameter $i$ denotes the ith microcell. Then we derive the number of mobile devices in all microcells and the number of mobile devices in the mcarocell

$$
\begin{aligned}
& N_{\text {MD,Micro }}=\sum_{i=1}^{N_{\text {Mirro }}} N_{i, \text { Micro }}, \\
& N_{M D, \text { Macro }}=N-N_{M D, \text { Micro }},
\end{aligned}
$$

The total bandwidth of ith microcell is $\mathrm{B}_{\mathrm{Mi}}$. Therefore, the bandwidth of each mobile device in ith microcell and the bandwidth of each mobile device in macro cell are

$$
\begin{aligned}
& B_{i, \text { Mi,user }}=\frac{B_{M i}}{N_{i, \text { Micro }},} \\
& B_{\text {MA,user }}^{\prime}=\frac{B_{M A}}{N_{M D, \text { Macro }}},
\end{aligned}
$$

We assume that the radius of the micro cell is D. The minimum required SNR at the edge of micro cell is SNRD. Due to the addition of microcells, the radius of macrocell will decrease to R'. So we derive the transmit power of microcell and macrocell as

$$
\begin{aligned}
& P_{t x, M i}=S N R_{D} \square \sigma^{2} \square K^{-1} \square D^{\lambda}, \\
& P_{t x, M A}^{\prime}=S N R_{D} \square \sigma^{2} \square K^{-1} \square R^{\prime \lambda}
\end{aligned}
$$

The SNR of mobile device which is $r$ meters away from micro base station and mcaro base station are

$$
\begin{aligned}
& S N R\left(r^{\prime}\right)=\frac{K \square P_{t x, M i} \square r^{r^{-\lambda}}}{\sigma^{2}}=S_{N}\left(\frac{D}{r^{\prime}}\right)^{\lambda}, \\
& \operatorname{SNR}\left(r^{\prime}\right)=\frac{K \square P_{t x, M a}^{\prime} \square r^{\prime-\lambda}}{\sigma^{2}}=S_{N}\left(\frac{R^{\prime}}{r^{\prime}}\right)^{\lambda},
\end{aligned}
$$

According to Shannon theorem, we derive the transmission rate as

$$
\begin{aligned}
& R_{M i}\left(r^{\prime}\right)=B_{M i, u s e r} \log _{2}\left(1+\operatorname{SNR}\left(r^{\prime}\right)\right), \\
& R_{M A}^{\prime}\left(r^{\prime}\right)=B_{M A, u s e r}^{\prime} \log _{2}\left(1+\operatorname{SNR}\left(r^{\prime}\right)\right),
\end{aligned}
$$

So the transmission duration of a mobile device at a distance of $r$ meters from ith micro and macro base station can be derived as

$$
\begin{aligned}
& t_{i, M i}\left(r^{\prime}\right)=\frac{S}{R_{M i}\left(r^{\prime}\right)}, \\
& t_{i, M A}^{\prime}\left(r^{\prime}\right)=\frac{S}{R_{M A}^{\prime}\left(r^{\prime}\right)},
\end{aligned}
$$


Then the average transmission duration of all mobile devices in ith microcell and macrocell can be expressed as

$$
\begin{aligned}
T_{i, M i}= & \frac{\sum_{i=1}^{N_{i, \text { Micro }}} t_{i, M i}\left(r^{\prime}\right)}{N_{i, \text { Micro }}}, \\
T_{M A}^{\prime}= & \frac{\sum_{i=1}^{N_{\text {MD,Macro }}} t_{M A}^{\prime}\left(r^{\prime}\right)}{N_{M D, \text { Macro }}},
\end{aligned}
$$

The total power consumption of mobile devices in ith microcell

$$
P_{i, M D, \text { Micro }}=N_{i, \text { Micro }}\left[P_{\text {work }} \frac{T_{i, M i}}{T}+P_{i d l e}\left[\left(1-\frac{T_{i, M i}}{T}\right)\right]\right. \text {, }
$$

And the power consumption of mobile devices in all microcells is given by

$$
P_{\text {total }, M D, \text { Micro }}=\sum_{i=1}^{N_{\text {Micro }}} P_{i, M D, \text { Micro }} \text {, }
$$

The power consumption of mobile devices in macrocell is

$$
P_{\text {total,MD,Macro }}=\left(N-N_{M D, \text { Micro }}\right)\left[P_{\text {work }} \frac{T^{\prime}{ }_{M A}}{T}+P_{\text {idle }} \llbracket\left(1-\frac{T^{\prime}{ }_{M A}}{T}\right)\right] \text {, }
$$

The total power consumption of mobile devices can be expressed as

$P_{\text {total }, M D}=P_{\text {total }, M D, \text { Micro }}+P_{\text {total }, M D, \text { Macro }}$

Then we calculate the power consumption of microcells. In a unit time, the static part of micro cell works at saturation power all the time, but the dynamic is not. So the dynamic part should be multiplied by a weight factor which is the downloading time.

$$
P_{B S, M i}=P_{s t a, M i}+\frac{T_{i, M i}}{T} \square P_{d y n, M i},
$$

So the power consumption of all microcells is given as follows

$$
P_{\text {total, } \text {,icro }}=\sum_{i=1}^{N_{\text {Micro }}} P_{B S, \text { Micro }} \text {, }
$$

We derive the decreased macrocell power consumption as

$$
P_{B S, M a}^{\prime}=N_{\text {Sec }} N_{P A}\left(\frac{P_{t x}^{\prime}}{\mu_{P A}}+P_{S P}\right)\left(1+C_{C}\right)\left(1+C_{P S}\right)
$$

So the total power consumption is given as follows

$$
P_{\text {total }}=P_{B S, \text { Macro }}^{\prime}+P_{\text {total,micro }}+P_{\text {total }, M D} \text {, }
$$

Which includes the decreased macrocell power consumption, the power consumption of all mobile devices and the power consumption of all microcells.

\section{Simulation and analysis}

To verify the accuracy of analytical results, a large number of simulations are provided in this section. The parameters of simulation are given in Table I. We usually consider four scenarios: macrocell only, one microcell per macrocell, three microcells per macrocell, and six microcells per macrocell. Each micro base station only contributes $1 / 3$ of its area to the coverage. The deployment of microcells are showed in Fig.1.As it turns out, several microcells are located at the edge of macrocell. We assume that $95 \%$ of the hexagonal area is covered either by macrocell or microcell. Both macrocell and microcell contribute to the coverage. Therefore, when a new microcell is added to the 
edge of a macrocell, the coverage of macrocell will decrease which leads to a lower transmit power and power consumption. In Fig.2, the radius of microcell is fixed at $15 \%$ of the radius of macrocell. It shows the relationship of area power consumption to different radius of macrocell. It is seen that deploying a varying number of microcells could reduce the area power consumption when the radius is larger than a threshold. However, it could increase the area power consumption for lower radius. So when the radius of macrocell is larger than $1900 \mathrm{~m}$, it is best to use six microcells per macrocell which will minimize the area power consumption.

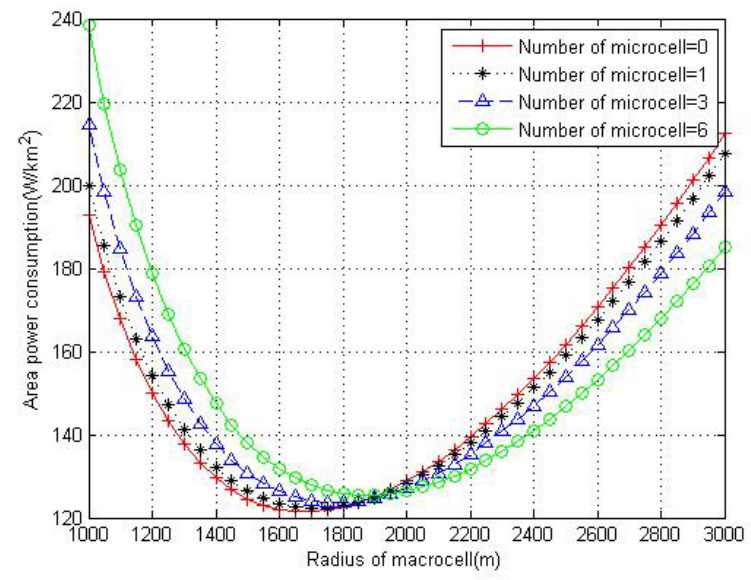

Fig. 2 Area power consumption over radius of macrocell

Fig. 2 shows that for certain radius of microcell, utilizing microcells decreases the area power consumption. However, the effect of different radii of microcells is not considered. Therefore, we fix the radius of microcell at $20 \%$ of the radius of macrocell in Fig.3. When the microcell size is larger, we see that utilizing microcells becomes a better strategy for bigger radius of macrocell. On the other hand, the area power consumption increase for smaller radius of macrocell. In this scenario, since larger microcells could cover a larger area, macro base stations can decrease more transmit power considerably while maintaining the coverage constraint.

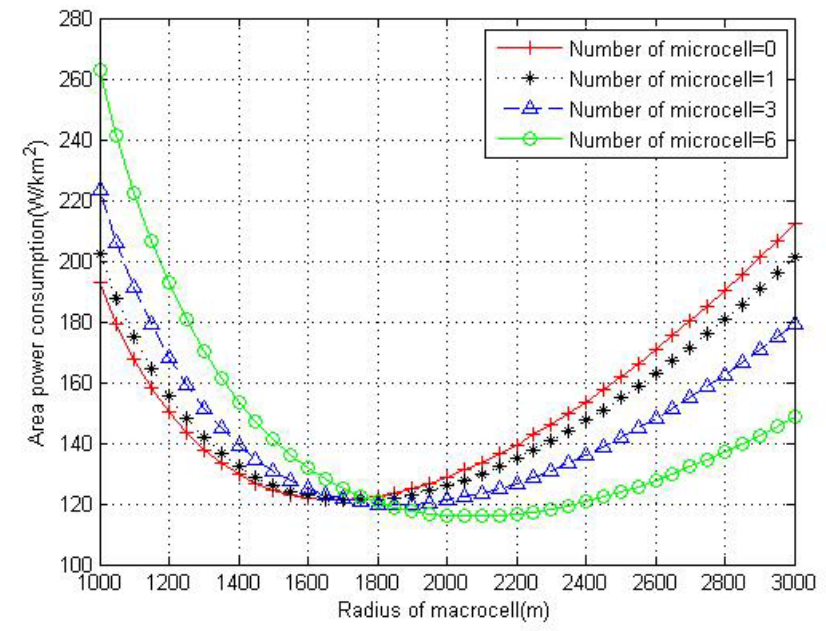

Fig.3 Area power consumption over radius of macrocell

Fig. 2 and Fig. 3 suggest that increasing the radius of microcells reduces the area power consumption. However, if we continue to increase the size of microcells, they no longer stay as microcells. In such a case, the micro base stations need to be as large and powerful as macro base stations. Then, they have to obey power consumption model of macrocells. Therefore, we cannot keep increasing the size of microcells.

In Fig. 4, we analysis the relationship of dynamic power consumption of mobile devices to the radius of microcells. It is seen that area power consumption decreases with the increase in the number and radiuses of microcells. On one hand, when a new microcell is added to the system, the capacity will increase, thus the time of downloading will reduce which leads to the reduction of dynamic power consumption of mobile devices. On the other hand, when the radiuses of microcells increase, 
more mobile devices will be included into the microcell. The channel condition of microcell is better than macrocell. Therefore, the power consumption will reduce with the increment of radius of microcells.

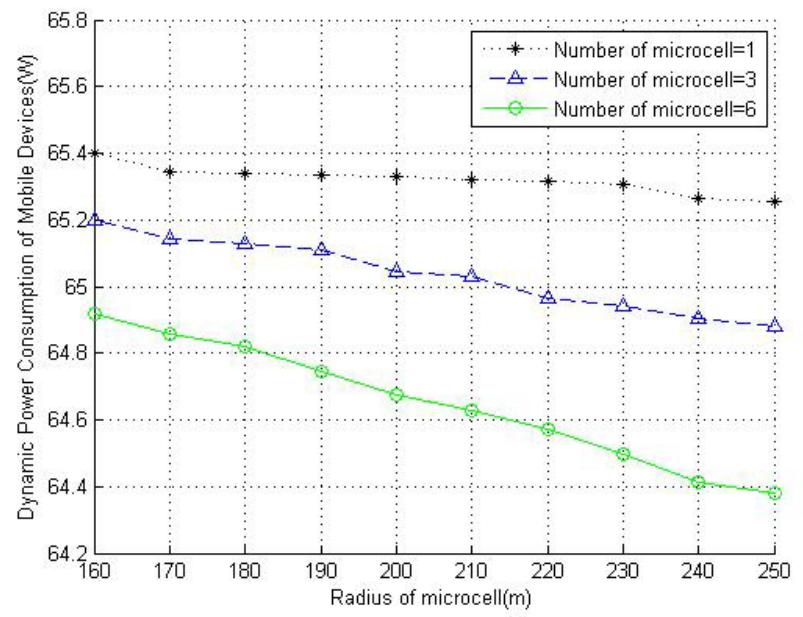

Fig. 4 Dynamic power consumption of mobile devices over radius of microcell

\section{Conclusion}

In this paper, all our analysis is based on the assumption that the coverage is fixed and the mobile devices are uniformly distributed throughout the area. We analyze the power consumption of both base stations and mobile devices in a more comprehensive perspective. We observe that both increasing the number of microcells and expanding the radius of microcells could reduce the area power consumption under certain conditions. Besides, we find it is possible to choose a certain number and radius of microcells to minimize the area power consumption.

Table I

SimULATION PARAMETERS

\begin{tabular}{|c|c||c|c|}
\hline \multicolumn{5}{|c|}{ Macrocell Parameters } \\
\hline \hline parameter & value & parameter & value \\
\hline \hline$N_{S e c}$ & 3 & $P_{S P}$ & $58 w$ \\
\hline$N_{P A}$ & 2 & $C_{C}$ & 0.29 \\
\hline$\mu_{P A}$ & $38 \%$ & $C_{P S}$ & 0.11 \\
\hline Bandwidth & $20 \mathrm{MHz}$ & Noise & $-160 \mathrm{dBm} / \mathrm{Hz}$ \\
\hline$S N R_{R}$ & $10 \mathrm{~dB}$ & Carrierfrequency & $2.0 \mathrm{GHz}$ \\
\hline \hline \multicolumn{5}{|c||}{ Microcell Parameters } \\
\hline \hline$C_{T X, s t a}$ & 0.8 & $P_{S P, s t a}$ & $15 \mathrm{w}$ \\
\hline$C_{t z, N L}$ & 0.04 & $P_{s p, N L}$ & $0.55 \mathrm{w}$ \\
\hline$\mu P A$ & $20 \%$ & $C_{P S}$ & 0.11 \\
\hline Bandwidth & $2 \mathrm{MHz}$ & Noise & $-150 \mathrm{dBm} / \mathrm{Hz}$ \\
\hline$S N R_{D}$ & $10 d B$ & Carrierfrequency & $2.0 \mathrm{GHz}$ \\
\hline \hline
\end{tabular}

\begin{tabular}{|c|c|c|c|}
\hline \multicolumn{2}{|c|}{$P_{\text {work }}$} & \multicolumn{2}{|c|}{$1.2 w$} \\
\hline \multicolumn{2}{|c|}{$P_{\text {idle }}$} & \multicolumn{2}{|c|}{$0.6 w$} \\
\hline \multicolumn{2}{|c|}{$S$} & \multicolumn{2}{|c|}{$100 k b i t$} \\
\hline \multicolumn{4}{|c|}{ Propagation Parameters } \\
\hline \multicolumn{2}{|c|}{ Macrocell } & \multicolumn{2}{|c|}{ Microcell } \\
\hline$\lambda$ & 4 & $\lambda$ & 2.6 \\
\hline$K$ & 7.081 & $K$ & 70.081 \\
\hline
\end{tabular}

\section{Acknowledgments}

The authors would like to express their thanks to the reviewers for their detailed suggestions. This work was supported by the National Natural Science Foundation of China (61372117). 


\section{References}

[1] R. Q. Hu, Y. Qian, S. Kota, and G. Giambene, "Hetnets - a new paradigm for increasing cellular capacity and coverage [guest editorial]," IEEE Wireless Communications, vol. 18, no. 3, pp. 8-9, 2011.

[2] H. Claussen, L. T. W. Ho, and F. Pivit, "Effects of joint macrocell and residential picocell deployment on the network energy efficiency," in IEEE International Symposium on Personal, Indoor and Mobile Radio Communications, 2008, pp. 1-6.

[3] Y. Hou and D. I. Laurenson, "Energy efficiency of high qos heterogeneous wireless communication network," in IEEE Vehicular Technology Conference Fall, 2010, pp. 1-5.

[4] T. Chen, H. Kim, and Y. Yang, "Energy efficiency metrics for green wireless communications," in International Conference on Wireless Communications and Signal Processing, 2010, pp. 1-6.

[5] F. Richter, A. J. Fehske, and G. P. Fettweis, "Energy efficiency aspects of base station deployment strategies for cellular networks," in IEEE Vehicular Technology Conference, 2009, pp. 1-5.

[6] O. Arnold, F. Richter, G. Fettweis, and O. Blume, "Power consumption modeling of different base station types in heterogeneous cellular networks," in Future Network and Mobile Summit, 2010, pp. 1-8.

[7] A. Goldsmith, "Wireless communications by andrea goldsmith, Cambridge university press, 2005 isbn 0-521-83716-2. Reviewed by stuart c. schwartz, princeton university,", 2006.

[8] L. Zhang, B. Tiwana, R. P. Dick, and Z. Qian, "Accurate online power estimation and automatic battery behavior based power model generation for smartphones," in Eighth Ieee/acm/ifip International Conference on Hardware/software Codesign and System Synthesis, 2010, pp. 105 114. 